\title{
Litiasis renal. El empedrado camino de la salud en Yucatán
}

\author{
Mario Basulto-Martínez $^{1^{*}}$ y Alfredo de J. Medina-Ocampo ${ }^{2}$ \\ ${ }^{1}$ Departamento de Urología; ${ }^{2}$ Dirección General. Hospital Regional de Alta Especialidad de la Península de Yucatán. Mérida, Yucatán, México
}

Muchas enfermedades, sobre todo aquellas altamente prevalentes en el mundo, han logrado llamar la atención de autoridades sanitarias, comunidad científica y sociedad en general. Tal es el caso de la diabetes, las enfermedades cardiovasculares y el cáncer; o bien enfermedades emergentes como el zika y la chikungunya. Resultado de esta notabilidad es que existan políticas públicas de salud, estrategias de cobertura de atención sanitaria, divulgación de avances científicos, campañas de promoción de la salud y, por tanto, una sociedad informada. Cuando se unen todos los componentes de esta fórmula, epidemiológicamente holística, su efectividad está probada, especialmente cuando es cosmopolita. Estas estrategias parecen congruentes con un mundo globalizado, sin embargo, al igual que en este escenario, existe una necesidad paralela del reforzamiento de las particularidades locales. Dicho de otra forma, en la globalización de la atención sanitaria y el desarrollo científico hacia los problemas de salud no se es equitativo con todos los padecimientos, o por lo menos no con la misma intensidad. Si una enfermedad es endémica solo para ciertas comunidades, dependerá de la capacidad política, económica y científica de estas para abordarlas, perdiendo esa fuerza de cohesión del «trabajo en equipo» $y$, por supuesto, solo las regiones que la padecen podrán dilucidar los factores locales que la perpetúan. Esto aplicado a la emergente economía mexicana, que enfrenta grandes retos en materia de salud, con insuficiente aporte del producto interior bruto a la ciencia y con un número potencialmente mejorable de investigadores, hace que podamos entender la plataforma de despegue para afrontar los temas complejos ef salud pública.

Por otra parte, hay algunas enfermedades que $\frac{\circ}{0}$ son del todo abordadas de manera asertiva, inclusojdentro de la comunidad médica. Tal es el caso de lälitiasis renal (LR). Cuando hemos tenido la oportunidad de dirigirnos a la sociedad en general para hablap de la LR y describirla como es realmente, el asombro y afirmación del desconocimiento previo son la regla lmpera el conocimiento del dolor ocasionado por ețócólico renoureteral y el enorme mito que hay alrededor del consumo de agua de mala calidad y los remedios herbolarios, pero es prácticamente nulo el conociimiento sobre las infecciones del tracto urinario acompañantes, los efectos deletéreos de la uropatía obstructiva, las elevadas tasas de recurrencia, la necesidad de̋ múltiples procedimientos invasivos diagnósticos y tểrapéuticos no exentos de complicaciones, la exposiésón repetida a radiación ionizante, el efecto de la đieta y estilo de vida en el riesgo de desarrollar LR, lạposibilidad de tratamientos preventivos y la posible evoolución a insuficiencia renal crónica que puede culmihar en tratamiento sustitutivo de la función renal o trašsplante renal.

Es muy probable que la mayor parte de la nunca haya visto una campaña de prevención
Date of reception: 11-10-2019

Date of acceptance: 21-10-2019

DOI: 10.24875/HMCM.19000236
*Mario Basulto-Martínez

E-mail: basultourologia@gmail.com 
cambio, en enfermedades cardiovasculares, diabetes y cáncer, entre otras, sería mucho más probable que evocasen unas cuantas. Sin juzgar si estas últimas son óptimas y suficientes, resulta evidente que la sociedad ha generado el constructo mental que representa a cada uno de estos ejemplos; pero es difícil el tratar de generar una imagen que represente dignamente aquella que sufre un paciente con LR.

Es razonable pensar que esta disparidad promocional se deba a la prevalencia o incidencia de las enfermedades, lo cual pareciera verdad pues las campañas de promoción mundiales y nacionales se enfocarán en las enfermedades más prevalentes, pero si vemos las particularidades regionales, el panorama cambia drásticamente, como lo es el caso de la LR en Yucatán.

\section{YUCATÁN Y LA ÉPOCA DE PIEDRA}

La literatura indexada apunta que, al menos desde hace 35 años, sabemos que Yucatán ocupa el primer lugar nacional en casos de LR. Los escasos y antiguos reportes estiman una prevalencia de aproximadamente el 10\% ${ }^{1,2}$; sin embargo, consideramos que podría ser mayor, lo que significa que, en Yucatán, la LR podría tener una prevalencia tan alta como la diabetes. En el Hospital Regional de Alta Especialidad de la Península de Yucatán (HRAEPY), la LR constituye el principal motivo de atención médica en todas las áreas, también es la principal causa de intervención quirúrgica, saturando la atención al ser el único centro de referencia en la Península de Yucatán y la región sur-sureste de México. Desde nuestra perspectiva, esto obliga a la comunidad médica y científica a tomar cartas en el asunto. Además, constituye un verdadero reto científico, capaz de despertar la curiosidad de cualquier investigador con vocación, a la vez que genera un enorme reto en las políticas públicas pertinentes.

Se ha demostrado que, para el desarrollo de la LR, la genética y el medio ambiente participan prácticamente en partes iguales ${ }^{3}$. La situación medioambiental de la Península de Yucatán es por demás propicia para el desarrollo de LR, pues cuenta con clima cálido, agua de mala calidad, pobreza, obesidad, alto consumo de refrescos embotellados y deshidratación crónica, entre otros. Sin embargo, estas características, en mayor o menor grado, pueden encontrarse fácilmente en otras entidades de México y el mundo, sin necesariamente reflejarse en una alta prevalencia de LR. Esto nös obliga a pensar en un componente genético.

Sabemos que México alberga un gran númerơde comunidades indígenas y grupos étnicos con trastondos genéticos parcialmente mezclados entre ellos. Én Yucatán se ha demostrado que la población mayämmestiza tiene un componente genético único ancestral que es diferente a aquel compartido por los demás ğ grupos étnicos; incluso se ha sugerido el "componentę genético Maya» ${ }^{4}$, lo que pudiera condicionar la interacción con el medio ambiente. Con esto en mente, sabemos que tenemos una población genéticamente distínta en un medio ambiente altamente propicio, el resultado es una altísima prevalencia de LR con un escaso dêsarrollo de conocimiento científico al respecto. En palabras, los médicos e investigadores en Yucátán tenemos el compromiso moral de sumar esfuerzôs para hacer frente a este complejo, antiguo y desafendido problema de salud pública.

\section{NUESTRO APORTE Y COMPROMISO}

Bajo esta perspectiva, hemos trabajado para sumar en la generación de evidencia que conduzca a làqueducción del problema y a la amplificación de su cobertura. En lo primero, hemos desarrollado proyectos dęinvestigación que abordan temas clínicos, epidemolốgicos, genómicos y de calidad de vida de los pacientes con $\mathrm{LR}$, en los que sus fases preliminares han traidg, resultados alentadores y que pronto podrán ser consỵltados con mayor hondura en revistas especializadaș. En lo último, en el HRAEPY, tras varios años de esfǘerzo y tenacidad en esta misma postura, nos hemos he ho aliados estratégicos que han sido vitales para. Aulestro proyecto, como el caso de la Fundación por lă Salud en Yucatán, A.C., con la que se fraguó un ambicicioso proyecto para la cimentación de la primera Clínida de Litiasis, con equipamiento técnico médico de última generación. Se integró un equipo multidisciplinārlo de trabajo que incluye actores en áreas y depenđencias de salud, investigadores de distintas adscripclores, especialistas de la Secretaría de Medio Ambienté local, antropólogos médicos del Instituto Nacional de Antropología e Historia y especialistas en temas regionales de agua a través del Comité Técnico de Aguas Subterráneas de la Zona Metropolitana de Mérida, Yücatán. Se firmaron acuerdos de colaboración para abarcar todos los posibles recovecos causales de la LR, y que 
en conjunto se erige como «Proyecto Tuunich» (Tuunich: piedra en lengua Maya), fungiendo como una iniciativa en salud para el diagnóstico, tratamiento, prevención, investigación y difusión de LR.

Estos esfuerzos son tangibles, pero aún nacientes. Continuamos en la búsqueda de la consolidación de este modelo de atención integral que cubra cada una de las aristas para este complejo problema de salud local, que es imposible lograr desde una individualidad o una sola institución, pues requiere la intervención de toda la sociedad para una solución de fondo y no remedios transitorios, tras más de 35 años de limitados esfuerzos. Parafraseando el proverbio, el emprendedor edifica con la piedra que tropezó. De igual modo, buscamos ser emprendedores y tomar la adversidad para hacer grandes cambios, buscar una solución de fondo y no seguir tropezando con la misma piedra, šno tomarlas y edificar un programa sólido que nos ⿸尸్rmita incidir de manera contundente y resolutiva en la a salud de la población que, está de más decirlo, puedè tomar generaciones.

\section{BIBLIOGRAFÍA}

1. Medina-Escobedo M, Zaidi M, Real de León E, Orozco-Rivadeneyra S. Prevalencia y factores de riesgo en Yucatán, México, para litiasis urinaria. Salud Pública Méx. 2002;44(6):541-5.

2. Gómez F, Reyes G, Espinosa L, Arellano H, Morales M, Gómez-B̃ Algunos aspectos epidemiológicos de la litiasis renal en México. Cir Ciruj. 1984:52:365-72.

3. Goldfarb DS, Fischer ME, Keich Y, Goldberg J. A twin study of genetic and dietary influences on nephrolithiasis: a report from the VietnamE Era Twin (VET) Registry. Kidney Int. 2005;67(3):1053-61.

4. Moreno-Estrada A Gignoux CR, Fernandez-López JC, Zakharia FESikora M, Contreras AV, et al. Human genetics. The genetics of Mexico recapitulates Native American substructure and affects biomedical traits. Science. 2014;344(6189):1280-5 